\title{
THE ELECTRONIC STRUCTURE AND BAND GAPS IN TRANSITION METAL COMPOUNDS
}

\author{
J. ZAANEN, G.A. SAWATZKY and J.W. ALLEN ${ }^{\dagger}$ \\ Physical Chemistry Department, Materials Science Center, University of Groningen, 9747 AG Groningen, The Netherlands
}

\begin{abstract}
A many body theory recently introduced for describing the electronic structure of rare earth metals is applied to $3 \mathrm{~d}$ transition metal compounds. The band gaps, their character and systematics, as well as various spectral distribution functions. are calculated and compared to recently obtained experimental data, for $\mathrm{NiO}$ and $\mathrm{NiS}$.
\end{abstract}

The electronic structure of $3 \mathrm{~d}$ transition metal compounds has been a controversial topic of discussion since 1937 when De Boer and Verwey [1] pointed out that the insulating behaviour of many of the compounds was inconsistent with the then available theory [2] for the electronic structure of solids. This problem was basically solved by Mott [3] and Hubbard [4] who showed that if the $\mathrm{d}-\mathrm{d}$ Coulomb interaction is larger than the one electron dispersional part of the band width a correlation gap will result with the Fermi level in the gap. These ideas have formed the basis for the understanding of a large range of physical properties of TM compounds of which the most important for our discussions are: the band gaps, the optical and magnetic properties. For the $\mathrm{Mn} \rightarrow \mathrm{Cu}$ oxides and halides these properties are semi-quantitatively well described by assuming a large $d-d$ Coulomb interaction which prevents polarity fluctuations in the $\mathrm{d}$ band and stabilizes a ground state with an almost integral number of $d$ electrons on each ion corresponding to that expected for an ionic compound. The deviation from ionicity is caused mainly by hybridization (covalency) with the anion $p$ band which involves a charge transfer energy. From the above follows that the local magnetic moment is given by that of an ion $\left(\mathrm{d}^{\prime \prime}\right)$ placed in the appropriate point group symmetry which in turn is governed by the Tanabe-Sugano [5] diagrams. Also the excitations in the $\mathrm{d}^{n}$ manifold are given by these diagrams and correspond to sharp optical transitions [6] observed for energies below the band gap. These excitations should be described as Frenkel excitons [7]. Important to note is that in the above description the transitional symmetry and therefore the $\mathrm{d}$ band dispersion has not entered. In fact many of the properties can be described by considering a TM ion as an "impurity" in the solid.

The translational symmetry enters in the description of the collective magnetic properties, generally successfully described by spin-only Hamiltonians with interatomic exchange interactions given by Andersons theory of superexchange [8] and the Goodenough-Kanamori rules [9]. These theories are also based on the assumption of large $\mathrm{d}-\mathrm{d}$ Coulomb interactions.

\footnotetext{
† XEROX Palo Alto Research Center. 3333 Coyote Hill Road. CA 94304, Palo Alto, USA.
}

The problems remaining concern mainly the nature of the band gap, the magnitude of the Coulomb interactions, and the nature of the states at the Fermi level of the metallic sulfides and some early TM oxides. In the numerous discussions on the nature of the band gap in insulators, $\mathrm{NiO}$ has played a central role as an example. The band gap could be of $d-d, O 2 p-T M 4 s, O 2 p-T M 3 d$ or TM3d-TM4s character as discussed by numerous authors [7,10-12]. Recent sophisticated local density functional band structure calculations obtain a $\mathrm{d}-\mathrm{d}$ gap of $0.3 \mathrm{eV}$ in $\mathrm{NiO}$ [13] although $\mathrm{FeO}$ and $\mathrm{CoO}$ are still metallic. That one obtains a gap at all is impressive but recent direct measurements $[14,15]$ on $\mathrm{NiO}$ show that the band gap is an order of magnitude larger. Also there is considerable evidence that the band gap in $\mathrm{Ni}$ compounds is of a charge-transfer $(\mathrm{O} 2 \mathrm{p}-\mathrm{Ni} 3 \mathrm{~d})$ rather than $\mathrm{d}-\mathrm{d}$ nature. This is suggested by Raman [16] experiments and also by the direct relation between the band gap and anion electronegativity [10]. The measured gaps are $\mathrm{NiS}(0 \mathrm{eV}), \mathrm{NiI}_{2}(1.7 \mathrm{eV})$ [17], $\mathrm{NiBr}_{2}(3.2 \mathrm{eV})$ [17]. $\mathrm{NiO}(4.3 \mathrm{eV})[14]$. On the other hand the band gaps in the early TM compounds $\mathrm{Ti}, \mathrm{V}$ are known to be of $\mathrm{d}-\mathrm{d}$ character [7].

In this paper we present a new [18,19] way of looking at the ground and excited states of TM compounds and arrive at a classification scheme which is consistent with available experimental data as well as known trends in the $\mathrm{d}-\mathrm{d}$ Coulomb interactions and anion electronegativities.

Our method is based on a broken (translational) symmetry calculation for the TM ions. This is equivalent to treating the $\mathrm{d}$ states of the TM as impurity states in an anion lattice. The advantage here is that the $\mathrm{d}-\mathrm{d}$ Coulomb interactions and the extremely important atomic term splittings of a $\mathrm{d}^{n}$ configuration can be taken into account explicitly using the very sophisticated techniques of configuration interaction involving both discrete and continuum states developed by Gunnarsson and Schönhammer [20]. They have shown that the near ground state properties (Kondo, valence fluctuation etc.) as well as the higher energy scale photoemission, inverse photoemission and core level spectroscopies can be understood within the same theoretical framework for rare earth metals and their compounds. Such a calculation is expected to be valid if the 
dispersional part of the band width is small as in $\mathrm{Ni}$ dihalides [21] and $\mathrm{NiO}[13](\approx 0.5 \mathrm{eV})$.

We could of course also break the anion translational symmetry ending up with a much used cluster approximation [18]. However, the anion $p$ band widths for the halides. oxides and sulfides are between 3 and 4 $\mathrm{eV}$ and therefore not negligible. Our calculation shows that the cluster approach is valid only if the band gaps are quite large $(>2 \mathrm{eV})$.

For the calculation we use an Anderson impurity Hamiltonian

$$
\begin{aligned}
H= & \sum_{m h} \epsilon_{k n} c_{k, n}^{+} c_{k n}+\sum_{m} \epsilon_{m} d_{m}^{+} d_{m}+\sum_{i j m} U(i j l m) d_{i}^{+} d_{j} d_{i}^{+} d_{m} \\
& +\sum_{m h n} V_{m k n}\left(d_{m}^{+} c_{k n}+c_{h n}^{+} d_{m}\right) \\
= & H^{\text {hew }}+H^{\text {imp }}+H^{\text {hybr }} .
\end{aligned}
$$

The first term describes the "host" band structure which in our case involve the anion $\mathrm{p}$ and cation $4 \mathrm{~s}, \mathrm{p}$ bands. The 2 nd and 3 rd terms ( $H^{\mathrm{imp}}$ ) describe the d-electronic structure of a TM ion in the point group symmetry of the solid including crystal field splittings and in $U$ the Slater integrals $F^{0}, F^{2}$ and $F^{4}$. The 4 th term describes the hybridization interaction between the "impurity" $d$ states and the host bands. In fig. 1 we show the various possible situations which could be encountered. The solid lines are shown for $H^{\text {hytr }}=0$ and the dotted lines indicate what happens to the redistribution of $\mathrm{d}$ weight upon switching on $H^{\text {hybr }}$. We point out that the states below $E_{1}$ represent the $N-1$ electron eigenstates (electron removal spectrum) and the states above $E_{\mathrm{F}}$. represent the $N+1$ electron eigenstates (electron addition spectrum). The large splitting in the $\mathrm{d}-\mathrm{d}$ weight $(\approx U)$ is due to the difference in the d ionization and affinity which, taking the Hunds rule ground state term in each case, is given by

$$
U=E\left(\mathrm{~d}^{n-1}\right)+E\left(\mathrm{~d}^{n+1}\right)-2 E\left(\mathrm{~d}^{n}\right) .
$$

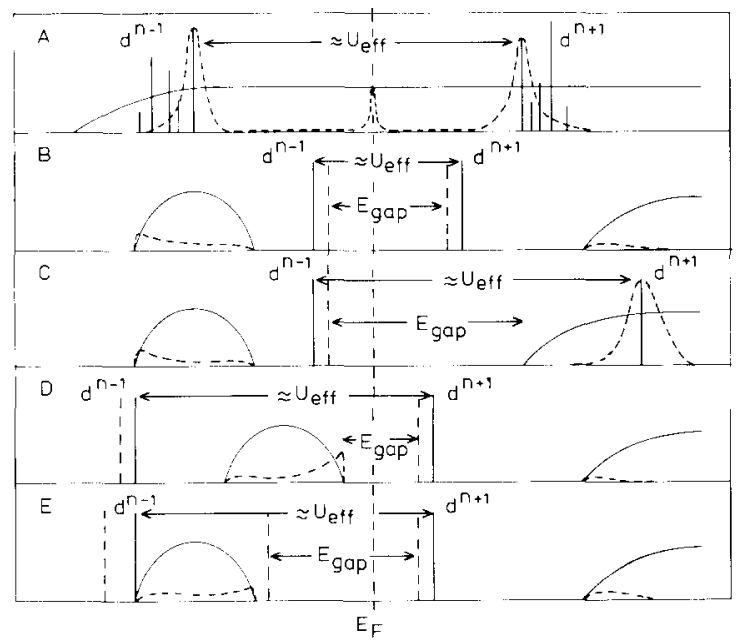

Fig. 1. An artists concept of the possible situations encountered for strongly correlated impurities in solids.
Defined in this way $U$ contains both the monopole $\left(F^{\prime \prime}\right)$ and the higher multipole $\left(F^{2} . F^{4}\right)$ Slater integrals of which $F^{0}$ is strongly screened in the solid whereas $F^{2}$ and $F^{4}$ remain close to the free ion values. $U$ therefore depends on the $\mathrm{d}^{\prime}$ ground state term. The conductivity gap is defined by

$$
E_{\text {gap }}=E_{\mathrm{l}}^{\text {solid }}(N-1)+E_{\mathrm{A}}^{\text {solid }}(N+1)-2 E_{\mathrm{g}}^{\text {solid }}(N) \text {. }
$$

Taking the ground state energy $\left(E_{\mathrm{g}}^{\text {solid }}(N)\right)$ for the $N$ electron system as zero, $E_{\text {gap }}$ is simply the minimum energy required to remove an electron plus that to add one electron. This is exactly what is measured by the onset of the photo- and inverse photo-electron spectra. respectively.

Fig. la shows what happens for a strongly correlated impurity in a metallic host. Here we also show the various possible term splittings of the $\mathrm{d}^{n} \quad\left(f^{n-1}\right)$ and $\mathrm{d}^{\prime \prime}+1\left(\mathrm{f}^{\prime \prime}+1\right)$ states. These are observed in the beautiful photo-inverse photo electron spectra of rare earth metals by Lang et al. [22]. Shown as dashed lines are the results of switching on $H^{\text {hybr }}$ causing a "virtual bound state" broadening and the possible formation of a Kondo resonance at the Fermi level as predicted theoretically $[20]$ and observed by photo and inverse photo emission [23].

The situations we can encounter in TM compounds are shown in figs. $1 b, c$ with a large, anion p-cation $4 s$, p, "host" band gap which is known to be $6-11 \mathrm{eV}$ [11]. Depending on the size of $U$ and the position of the $\mathrm{d}^{\prime \prime}{ }^{1} \mathrm{~d}^{\prime+1}$ states relative to the "host" bands the gap can be either anion p-TMd, TMd-TMd, TMd-TM4s, or even anion $\mathrm{p}-\mathrm{TM} 4 \mathrm{~s}$.

The most interesting cases for the late TM compounds are shown in figs. $1 d$ and $e$. Here the anion $p$ band lies in the $d-d$ correlation gap forming a charge transfer gap (fig. 1d). If the $\mathrm{d}^{n-1}$ state is close to or in the anion p band (fig. 1e) a "bound state" can be pushed up, out of the band by $H^{\text {hybr }}$ with a strongly mixed character. If the charge transfer energy (anion $\mathrm{p}-\mathrm{d}^{n+1}$ ) is also small this gap can close forming a metal but with a peak at the Fermi level very reminiscent of a Kondo resonance. The zero gap case of fig. 1d corresponds to a p type metal (CuS) and the zero gap case of fig. $1 \mathrm{c}$ is close to reality for NiS as shown below.

The problem in the calculation is a proper treatment of $H^{\text {hybr }}$. To do this exactly we neglect the $\mathrm{d}-4 \mathrm{~s}$ hybridization both because the $4 \mathrm{~s}$ band lies high in energy and also because the $\mathrm{d}-4 \mathrm{~s}$ mixing is expected to be small. In fig. 2 we show the basis configurations used. For the ground state calculation (center of fig. 2) we take the ionic configuration ( $H^{\text {hybrid }}=0$ ) as ansatz (i.e. $\mathrm{d}^{8}\left({ }^{3} \mathrm{~A}_{2 \mathrm{~g}}\right)$ for $\mathrm{Ni}^{2+}$ in $\mathrm{O}_{\mathrm{h}}$ symmetry). For $\mathrm{Ni}^{2+}$ the calculation involves a 2 -hole problem which can be solved exactly as shown for the equivalent impurity Auger spectrum calculation [24]. A similar calculation for the other irreducible representations spanned by $2-\mathrm{d}$ holes would yield the optical spectrum. To determine 


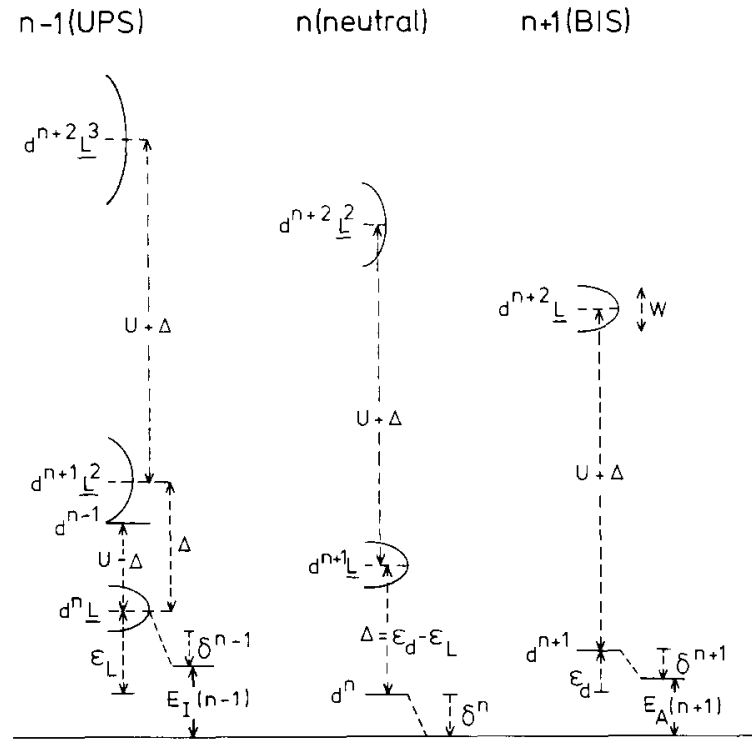

Fig. 2. Diagram showing the various states and parameters used in the calculation.

the band gap we also do the calculation with one electron removed (left hand side) and one electron added (right hand side) which again should be done for every irreducible representation of the point group. The lowest energy states of these relative to the ground state energy are a direct measure of the band gap.

The parameters used in the calculation are also indicated in fig. 2. For example for divalent $\mathrm{Ni}$ compounds the charge transfer energy $(\Delta)$ is given by

$\Delta=\left(E\left(\mathrm{~d}^{9} \underline{\mathrm{L}}\right)+E\left(\mathrm{~d}^{8}\right)\right)=\epsilon_{\mathrm{d}}-\epsilon_{\mathrm{L}}$,

measured from the center of the anion $p$ band $(L$ is a ligand hole).

Note that the $N-1$ particle states in fig. 2 have been drawn for $U>\Delta, V=W / 2$ where $W$ is the anion $\mathrm{p}$ band width, as suggested by Fujimori [18] for $\mathrm{Ni}$ compounds.

Switching on $H^{\text {hybr }}$ now mixes the various configurations within each diagram resulting in a very complicated spectrum of discrete, bound and shifted continuum states. For the band gap calculations we are only interested in the lowest energy state for each case which is in all cases shifted down by $H^{\text {hybr }}$ as indicated in fig. 2 by $\delta^{x}$. In terms of our parameters and the hybridization shifts $\left(\delta^{x}\right)$ the band gap is

$$
\begin{aligned}
& E_{\text {gup }}=\Delta-W / 2+2 \delta^{n}-\delta^{n+1}-\delta^{n-1} \quad(U>\Delta-W / 2) \text {, } \\
& E_{\text {gup }}=U+2 \delta^{n}-\delta^{n+1}-\delta^{n-1} \quad(U<\Delta-W / 2) \text {, }
\end{aligned}
$$

with $W$ as the anion $\mathrm{p}$ band width and with $\delta^{n-1}$ always measured from the lowest energy state of the system with $H^{\text {hybr }}$ set to zero. We see immediately from this that the gap follows the charge transfer energy for $U<\Delta$ as observed in divalent $\mathrm{Ni}$ compounds.
Note that to obtain the generally used picture with the $N-1$ particle states drawn below $E_{\mathrm{F}}$ and the $N+1$ particle states above $E_{\mathrm{F}}$ we have to rotate the $N-1$ particle spectrum of fig. 2 about the $N$ particle ground state line. This requires knowledge of $\delta^{\prime \prime}$ and can cause some problems in determining $U$ directly from photoemission and inverse photoemission experiments.

The details of the calculations, which follow procedures outlined by Gunnarsson and Schönhammer [20] will be published elsewhere [25]. As parameters we used $W=3 \mathrm{eV}$ and $V=1 \mathrm{eV}$. The calculation has been done for $n=10,9,8,0,1,2,3$ corresponding to $\mathrm{Cu}^{1+}, \mathrm{Cu}^{2+}$, $\mathrm{Ni}^{2+}, \mathrm{Sc}^{3+} \cdot \mathrm{Ti}^{3+}, \mathrm{V}^{3+}$ but we find that the results are rather insensitive to $n$ being determined more by $U, \Delta$ and $V$.

The energy gaps are shown in fig. 3 as a function of $\Delta$ and for various values of $U$. We notice that for $U=0$ the gap is always zero. This will be the case in any calculation in which the exchange and correlation are replaced by an effective one particle potential. Upon taking into account the translational symmetry of the TM ions a small gap may occur because of the $\mathrm{d}$ band dispersional relations. We also see that for $U$ large $(U>\Delta$ ) the gap is proportional to $\Delta$ and goes to zero for $\Delta<W / 2$ resulting in a metallic ground state. For $U \ll \Delta$ (right hand side) the gap is proportional to $U$ as in a Mott-Hubbard system. Next to these extreme cases there is an interesting region for $U \approx \Delta \pm W / 2$. In this region the $\mathrm{d}^{\prime \prime-1}$ state lies inside or close to the edges of the $\mathrm{d}^{\prime} \mathrm{L}$ continuum. The $N-1$ particle ground state is then determined by a bound state pushed out of the $\mathrm{d}^{n} \mathrm{~L}$ continuum due to the hybridization with the $\mathrm{d}^{n-1}$ discrete state. This bound state will broaden into a narrow band if we include the translational symmetry of the TM ions and contains both $\mathrm{d}^{n} \underline{\mathrm{L}}$ and $\mathrm{d}^{n-1}$ character. It is important to note that this bound state can occur for each IR spanned by the $n-1 \mathrm{~d}$ electrons and will therefore exhibit the same kind but somewhat reduced multiplet splittings. This was demonstrated nicely in the

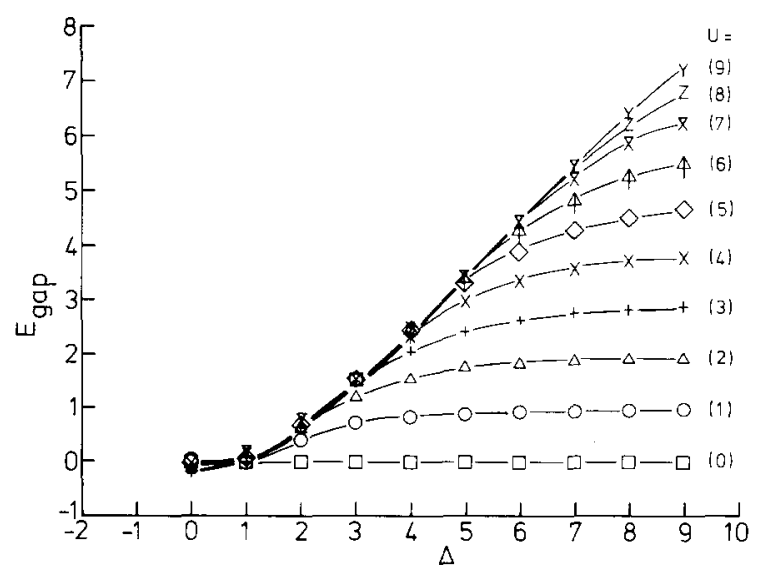

Fig. 3. The calculated band gap as a function of $\Delta$ for various values of $U$ and for $W=3 \mathrm{eV}$ and $V=1 \mathrm{eV}$. 
cluster calculations of Fujimori et al. [18].

A clear demonstration of the success of the above theory is not only the band gap systematics but also its success in describing the photo and inverse photoemission data which are a direct measure of the $N-1$ and $N+1$ particle spectral distributions. In fig. 4 we show two recently studied examples $\mathrm{NiO}[14]$ and the metallic NiS [26]. The assignment scheme at the top is done using a cluster theory approach for NiO. At the high photon and electron energies used the measured spectral weight is mainly that for $\mathbf{d}$ electron removal and addition. Inverse photoemission has also been done at lower energies $(10 \mathrm{eV})$ by Hufner et al. $[15,17]$.

For $\mathrm{NiO}$ we see the gap of $4.3 \mathrm{eV}$ and the just discussed bound state as the first photo ionization peak. The arrows drawn between the NiO and NiS data show the shifts of the structures seen due to a lower $\Delta$ and also a lower $U$. We notice the large total d electron spectral weight spread of about $30 \mathrm{eV}(-10$ to $+18 \mathrm{eV})$ for $\mathrm{NiO}$ and $15 \mathrm{eV}$ for $\mathrm{NiS}$. It is this large spectral weight spread as well as the band gaps and peaks positions which is a challenge to describe theoretically.

The d electron photo (inverse) emission spectrum is given by

$I_{\text {phortexinv }} \propto \operatorname{Im}\left\langle\Psi_{\mathrm{g}}\left|\mathrm{d}^{+}(\mathrm{d}) \frac{1}{z-H}\left(\mathrm{~d}^{+}\right) \mathrm{d}\right| \Psi_{\mathrm{g}}\right\rangle$.

$\Psi_{\mathrm{g}}$ is the ground state wave function and is of the form

$$
\begin{aligned}
\Psi_{g}\left({ }^{3} \mathrm{~A}_{2 g}\right)= & A\left|\mathrm{~d}^{8}\left({ }^{3} \mathrm{~A}_{2 g}\right)\right\rangle+\sum_{k} \alpha_{k}\left|\mathrm{~d}^{9 g} \underline{k}\left({ }^{3} \mathrm{~A}_{2 g}\right)\right\rangle \\
& +\sum_{k k^{\prime}} \beta_{k k^{\prime}}\left|\mathrm{d}^{10} \underline{k} \underline{k}^{\prime}\left({ }^{3} \mathrm{~A}_{2 g}\right)\right\rangle .
\end{aligned}
$$

with the coefficients determined by the above mentioned ground state calculation.

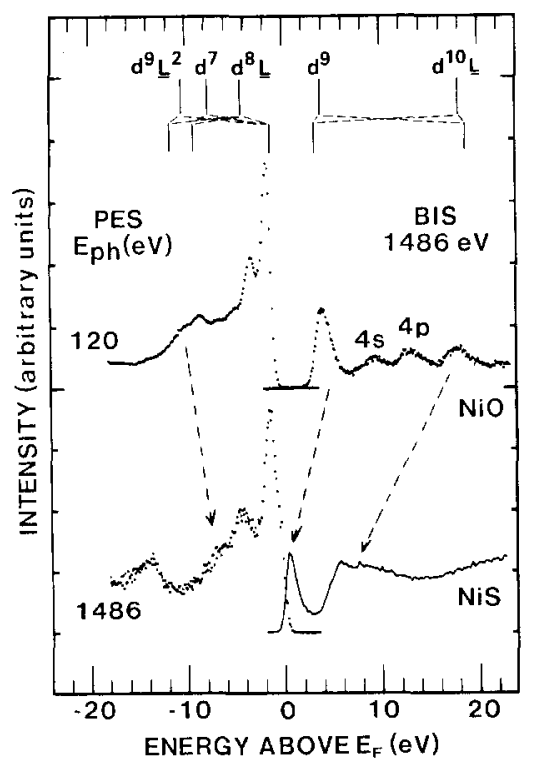

Fig. 4. Photo and inverse photoemission spectra of $\mathrm{NiO}$ and NiS. The bar diagram shows the assignments according to a cluster calculation.
To indicate the spectral distributions of the $N-1$ and $N+1$ basis states of fig. 2 upon including $H^{\text {hibr }}$ we show in fig. $5 \mathrm{a}$ and $c$ the imaginary parts of the Green's functions (e.g. $\sum_{k}\left\langle\mathrm{~d}^{8} \underline{k}|1 /(Z-H)| \mathrm{d}^{\mathrm{x}} \underline{k}\right\rangle=$ $G 8 \mathrm{~L}$ ) entering the $N+1$ electron spectrum. We see indeed the appearance of a bound state determining the first ionization state in both $\mathrm{NiO}$ and $\mathrm{NiS}$. In $\mathrm{NiO}$ this state has substantial $d^{7}$ as well as $d^{8} \underline{L}$ character. For NiS the lowest ionization state at the Fermi level is a mixture of the electronic configurations $d^{7}, d^{8} \underline{L}$ and $d^{9} L^{2}$. This state together with the lowest affinity state of $d^{9}$ and $d^{10} \underline{L}$ character and the ground state of $d^{8}, d^{y} \underline{L}$ form a sharp structure at $E_{\mathrm{F}}$, which is very reminiscent of a Kondo resonance. The exact nature of this state is $\mathrm{NiS}$ is very sensitive to the choice of $\Delta, V$ and $U$ and therefore to the lattice parameters which may indicate a mechanism for the phase transitions observed in NiS [28].

The calculated spectra are shown in figs. $5 b$ and $d$. The energy spread is the same as in figs. a and $c$ but now we take into account the ground state wave functions in the above equations. We notice that although the first ionization state in $\mathrm{NiO}$ has a lot of $\mathrm{d}^{8} \mathrm{~L}$ character it still has a very large spectral weight for $d$ electron removal consistent with photon energy dependent measurements [29]. This is a direct result of the constructive interference for the lowest energy state between the various ionization channels from the mixed ground state (i.e. $d^{8} \rightarrow d^{7}, d^{9} \underline{L} \rightarrow d^{8} \underline{L}, d^{10} \underline{L}^{2} \rightarrow d^{9} \underline{L}$ ). This is a general tendency as pointed out by Gunnarson and Schönhammer [20]. The theoretical spectra were calculated for only one of the many IR's for the $n-1$ electron spectrum and therefore do not show the splitting in the first ionization peaks observed experimentally.

We now finally come to a classification scheme based on the size and nature of the band gaps shown in fig. 4 . We realize that $\Delta$ is roughly proportional to the anion electron negativity for a given TM. We also realize that $U$ decreases in going from $\mathrm{Cu}$ to $\mathrm{Ti} \mathrm{com}$ pounds from about $8-9 \mathrm{eV} \mathrm{[30]} \mathrm{to} \mathrm{about} 1$ or $2 \mathrm{eV}$.

In fig. 6 we show a classification scheme on a $U$ vs. $\Delta$ plot. The solid line is the semi-conductor-metal separation line which we have drawn for our calculated gap at $0.5 \mathrm{eV}$ to take some account of the TM metal translation symmetry which will broaden all our states somewhat. The dashed line shows where our calculated gap goes to zero. The regions marked in fig. 6 follow directly from fig. 3 and the above discussion on the nature of the band gap. A is a semiconductor region with a $d-d$ correlation gap. Many of the early TM compounds belong in this region or to the region marked $\mathrm{C}$ corresponding to $\mathrm{d}$ band metals. Materials in $\mathrm{B}$ are also semiconductors but now with a charge transfer gap. Many of the late TM oxides and halides belong to this region, although the more ionic compounds like $\mathrm{CuF}_{2}$, $\mathrm{NiF}_{2}, \mathrm{NiO}, \mathrm{NiCl}_{2}$ belong to the region $\mathrm{AB}$ in which the 


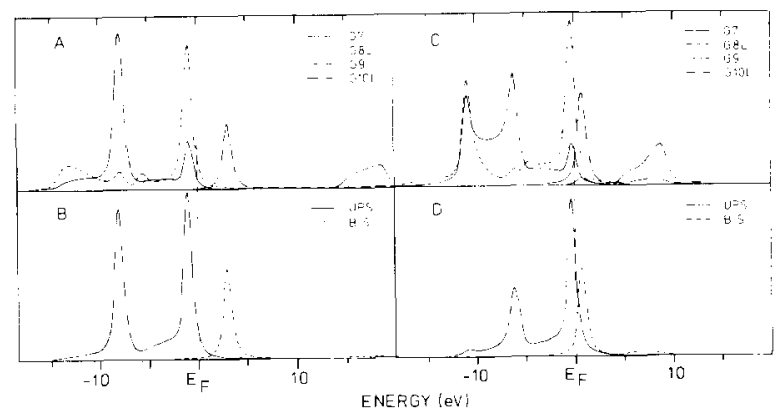

Fig. 5. A and $C$ show the imaginary parts of the $N-1$ and $N+1$ particle Green's functions as defined in the text for $\mathrm{NiO}$ $(\Delta=6, U=8, V=2)$ and $\mathrm{NiS}(\Delta=2, U=4, V=2)$ in both cases $W=4 \mathrm{eV}$. B and $\mathrm{D}$ are the corresponding photo- and inverse photoemission spectra for $\mathrm{NiO}$ and $\mathrm{NiS}$ respectively. $\mathrm{A}$ broadening of $1.4 \mathrm{eV}$ ( $\mathrm{fwhm}$ ) was used in all cases.

gap is determined by the strongly mixed bound state as discussed above. The regions marked $C^{\prime}, C^{\prime} D$ and $D$ describe metals. In $\mathrm{D}$ the Fermi level lies in the anion $\mathrm{p}$ band resulting in hole conduction as in many $\mathrm{Cu}$ sulfides and selenides. The region $C^{\prime} D$ is rather special because here the Fermi level is determined by the "bound state" discussed above with a very high density of states which however is of strongly mixed character. We believe that NiS, CoS and perhaps FeS belong to this region.

In our opinion the described calculation forms a good basis for classification of TM compounds. The parameters used can be determined by comparing calculated and experimental photo and inverse photoemission as well as optical spectra. In future papers we show that the same theory can also be used to describe core level spectroscopies. Aside from the extension of the calculation to include all irreducible representations of the point group the major challenge remaining is to

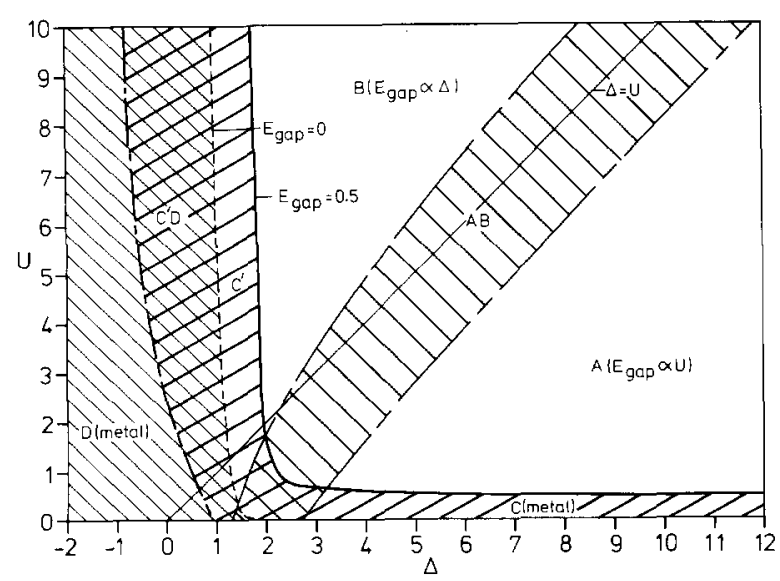

Fig. 6. A classification scheme based on the calculation described in the text. The various regions are explained in the text. This diagram is calculated for $W=3$ and $V=1 \mathrm{eV}$. find a way of incorporating the TM translation symmetry. This is of utmost importance for an understanding of the collective magnetic and transport properties. In this regard we believe that the described theory forms a basis for describing the nature of the quasi particles which could be used to incorporate the TM translation symmetry.

One of the authors (G.A.S.) would like to thank the XEROX Corporation for their hospitality during his stay at which time the ideas for this work were conceived. This work was supported in part by the Netherlands Foundation for Chemical Research (SON) with financial aid from the Netherlands Organization for the Advancement of Pure Research (ZWO).

[1] S.H. de Boer and E.J.W. Verwey, Proc. Phys. Soc. A49 (1937) 59.

[2] A.H. Wilson. Proc. Roy. Soc. A133 (1931) 458.

[3] N.F. Mott, Proc. Phys. Soc. A62 (1949) 416.

[4] J. Hubbard, Proc. Roy. Soc. A276 (1963) 238

[5] Y. Tanabe and S. Sugano, J. Phys. Soc. Japan 9 (1954) 766.

[6] J.W. Allen, in: Magnetic Oxides, ed. D.J. Craik (Wiley, London, 1975) p. 394.

[7] N.F. Mott, in: Metal-Insulator Transitions (Taylor and Francis, London, 1974).

[8] P.W. Anderson, Phys. Rev. 115 (1959) 2.

[9] J.B. Goodenough, Phys. Rev. 100 (1955) 564; J. Phys. Chem. Sol. 6 (1958) 287. J. Kanamori, J. Phys. Chem Solids 10 (1959) 87.

[10] J.A. Wilson, Advan. Phys. 21 (1972) 143.

[11] B. Koiller and L.M. Falicov, J. Phys. C. 7 (1974) 299.

[12] D. Adler and J. Feinleib, Phys. Rev. B2 (1970) 3112.

[13] K. Terakura, A.R. Williams, T. Oguchi and J. Kubler, Phys. Rev. Lett. 52 (1984) 1830

[14] G.A. Sawatkzy and J.W. Allen, Phys. Rev. Lett. 53 (1984) 2339.

[15] S. Hufner, J. Osterwalder, T. Riesterer and F. Hulliger, Solid State Commun. 52 (1984) 793.

[16] R. Merlin, T.P. Martin, A. Polian, M. Cardona, B. Andlauer and D. Tannhauser, J. Magn. Magn. Mat. 9 (1978) 83.

[17] C.R. Ronda and G.J. Arends, private communication.

[18] A. Fujimori and F. Minami, Phys. Rev. B30 (1984) 957.

[19] J. Zaanen, G.A. Sawatzy and J.W. Allen, to be published.

[20] O. Gunnarsson and K. Schönhammer, Phys. Rev. Lett. 50 (1983) 604; Phys. Rev. B28 (1983) 4315, B31 (1985) 4815.

[21] R. Coehoorn, private communication.

[22] J.F. Lang, Y. Bear and P.A. Cox, J. Phys. F 11 (1981) 121.

[23] J.W. Allen, Phys. Rev. B28 (1983) 5347.

[24] M. Vos, D. van der Marel and G.A. Sawatzky, Phys. Rev. B24 (1984) 3073.

[25] J. Zaanen and G.A. Sawatzky, to be published.

[26] J.A. Allen, J. Zaanen and G.A. Sawatzky, to be published.

[27] S. Hufner, T. Riesterer and F. Hulliger, Solid State Commun. 54 (1985) 689.

[28] D.B. McWhan, M. Marezio, J.P. Remeika, P.D. Dernier, Phys. Rev. B5 (1972) 2552.

[29] D.E. Eastman and J.F. Freeof, Phys. Rev. Lett. 34 (1975) 395.

[30] G. van der Laan, G.A. Sawatzky, C. Haas and H.W. Myron, Phys. Rev. B20 (1979) 4287. 